\title{
POSS Grafting on Polyethylene and Maleic Anhydride-Grafted Polyethylene by One-Step Reactive Melt Mixing
}

\author{
ELISABETTA MORICI, ALBERTO DI BARTOLO, ROSSELLA ARRIGO, \\ NADKA TZANKOVA DINTCHEVA \\ Dipartimento di Ingegneria Civile, Ambientale, Aerospaziale, dei Materiali, Università di Palermo, Viale delle Scienze, Ed. 6, 90128 Palermo, \\ Italy \\ Correspondence to: Nadka Tzankova Dintcheva; e-mail: nadka.dintcheva@unipa.it.
}

Received: July 29, 2015

Accepted: January 5, 2016

\begin{abstract}
This study reports the one-step reactive melt mixing preparation of organic-inorganic hybrid based on maleic anhydride-grafted polyethylene and 3-(2-aminoethyl)aminopropyl-heptaisobutyl substituted polyhedral oligomeric silsesquioxane, (NPOSS), as well as on low-density polyethylene and allyl-heptaisobutyl-POSS (1POSS) in dicumyl peroxide presence, which is believed to activate the unsaturation of the reactive functional group of POSS itself. The successful grafting of POSS molecules onto polymeric backbones was probed through rheological and spectroscopic analysis. Grafting of POSS molecules enhances their dispersion in the polymeric matrix as shown by morphological analysis. Moreover, the rheological analysis of the organic-inorganic hybrids reveals a solid-like behavior, due to the formation of a network related to the interactions between grafted POSS molecules. The homogeneous dispersion of POSS molecules along with the strong interaction between polymeric segments and the nanoparticles influences the calorimetric and mechanical properties of the obtained hybrids. (c) 2016 Wiley Periodicals, Inc. Adv Polym Technol 2016, 00, 21673; View this article online at wileyonlinelibrary.com. DOI 10.1002/adv.21673
\end{abstract}

KEY WORDS: Functionalization of polymers, Polyethylene (PE), Reactive processing, Rheology, Structure-properties relations

\section{Introduction}

$\mathbf{T}$ he possibility to properly combine properties of organic and inorganic components for materials design and processing is a challenging issue from scientific and industrial point of view. Indeed, hybrid materials are suitable for advanced material project, finding applications in several fields such as optics, electronics, biotechnology, medicine, functional smart coatings, fuel and solar cells, catalysts, and sensors. ${ }^{1}$ A particular focus is on hybrid materials based on organic polymeric matrices since, as known, polymers are extensively used in industry and in everyday life because of their broad range of properties, mainly lightweight and ductility. ${ }^{2}$ In the past years, the use of polyhedral oligomeric silsesquioxane (POSS) nanoparticles has been demonstrated to be an effective method in the design of hybrid polymeric materials with better thermal and mechanical properties. $^{3-6}$ Typical and most used POSS molecules are characterized by the general formula $\left(\mathrm{RSiO}_{1.5}\right)_{8}$ and involve a rigid and cubic inorganic silica core of about $0.3-0.4 \mathrm{~nm}$, surrounded by hydrogen and/or organic group. In particular, one of the members of POSS family consists of a $\mathrm{Si}_{8} \mathrm{O}_{12}$ core bounded to seven organic groups on the corners, promoting solubility in polymeric matrices, and one unique group at the final corner which is used as a reactive site of polymerization or grafting., ${ }^{4,7}$
Moreover, the use of seven same organic groups instead of eight permits to obtain a better dispersion in the matrix due to the asymmetry of the molecule that reduces autoaggregation phenomena, as known in the literature. ${ }^{8}$ The POSS incorporation in polymer matrices by using physical blending (no reactive melt mixing) is a fast, adaptable, and profitable way to obtain hybrid nanocomposites, but the successful dispersion of POSS depends on the interactions, which are Van der Waals, hydrogen, and polar bonding, between POSS molecules and polymers. ${ }^{9-13}$ Since a uniform POSS incorporation into some polymers leads to enhancements in thermal stability, mechanical properties, and reduces flammability, it is important to avoid aggregation of the nanoparticles during melt mixing that, often, is a serious problem in POSS/polymer nanocomposites formulation. ${ }^{14}$ The formation of a chemical bond between POSS nanoparticles and polymer backbone is an alternative route to successful incorporation, ${ }^{15}$ and, usually, POSS-based homogeneous hybrid polymeric materials are prepared through the one-pot copolymerization method, i.e., POSS monomers and organic monomers mixed together prior to the polymerization process. ${ }^{16,17} \mathrm{How}$ ever, this last approach involves procedures that limit the commercialization of the resulting materials. Recently, grafting of POSS into the polymeric matrix by reactive processing, that is a solvent-free method for the chemical modification of existing polymers, has been thought as an advisable solution to solve 
[3-(2-Aminoethyl)amino]propyl-Heptaisobutyl POSS (named here NPOSS)

\begin{tabular}{lll}
\hline & & Molecular formula \\
Molecular weight & & $\mathrm{C}_{33} \mathrm{H}_{76} \mathrm{~N}_{2} \mathrm{O}_{12} \mathrm{Si}_{8}$ \\
917.65 \\
\hline
\end{tabular}

issues related to the nanofillers' incorporation into polymer systems, maintaining ease of processing, versatility, and costeffectiveness. ${ }^{18-20}$ In particular, the dispersion of the nanoparticles in the polymeric matrix as well as polymer/nanofiller interactions is expected to be improved by the use of POSS molecules bearing functional groups that can interact with reactive groups grafted on the polymeric backbone during a one-step melt blending ${ }^{21,22}$ or introducing reactive groups onto the POSS molecules that can be properly activated during processing to attach them in the polyolefin chains. ${ }^{23}$ Therefore, inorganic POSS molecules containing a reactive monofunctionality may be the ideal nanofiller for use in the construction of organic-inorganic hybrid nanomaterials by one-step reactive melt mixing.

In this study, we explored two different preparation approaches based on reactive melt processing to formulate an organic-inorganic polymeric hybrid. A polyethylene (PE) containing side-chain-tethered caged POSS has been obtained using maleic anhydride-grafted polyethylene (PEgMA) and an amino ethyl-amino propyl-heptaisobutyl substituted POSS, whereas a second hybrid has been produced using PE and allylheptaisobutyl-POSS together with a small amount of dicumyl peroxide (DCP) used as an initiator. The successful grafting was assessed by Soxhlet extractions, rheological and spectroscopical analysis. Moreover, both hybrids were fully characterized through morphological, calorimetric, and mechanical analysis.

\section{Experimental}

\section{MATERIALS}

Low-density PE was produced by Versalis (Milan, Italy) under the trade name "Riblene FC 30 " with a melt flow index $0.27 \mathrm{~g} /$ $10 \min \left(190^{\circ} \mathrm{C}, 2.16 \mathrm{~kg}\right)$.
PEgMA was produced by Auserpolimeri (Lucca, Italy) under the trade name Compoline CO/LL with a melt flow index of $1.2 \mathrm{~g} / 10 \mathrm{~min}\left(190^{\circ} \mathrm{C} / 2.16 \mathrm{~kg}\right)$ and density $0.9 \mathrm{~g} / \mathrm{cm}^{3}$.

[3-(2-Aminoethyl)amino]propyl-heptaisobutyl substituted polyhedral oligomeric silsesquioxane (NPOSS) and allylheptaisobutyl substituted polyhedral oligomeric silsesquioxane (1POSS) were produced by Hybrid Plastics (Hattiesburg, MS, USA). Structure, molecular weight, and empirical molecular formula of both POSS molecules are reported in Table I.

DCP, supplied by Sigma-Aldrich (Milan, Italy), has a linear formula $\left.\mathrm{C}_{6} \mathrm{H}_{5} \mathrm{C}\left(\mathrm{CH}_{3}\right)_{2}\right]_{2} \mathrm{O}_{2}$ and molecular weight $270.37 \mathrm{~g} / \mathrm{mol}$.

\section{PREPARATION AND CHARACTERIZATION}

POSS-based hybrid nanocomposites were prepared by reactive melt blending using a Brabender PLE-330 internal mixer (Duis- burg, Germany) at $180^{\circ} \mathrm{C}$ for $6 \mathrm{~min}$ at $60 \mathrm{rpm}$. POSS loading was $3 \mathrm{wt} \%$ to the samples' total weight. The DCP was used as an initiator for the reaction between 1POSS molecules and PE matrix. The DCP amount, i.e., $0.3 \mathrm{wt} \%$ to the samples' total weight, was chosen as a result of preliminary tests to check the minimum amount for POSS grafting. Neat PE, PEgMA, as well as PE/DCP and PE/1POSS samples were processed under the same conditions for sake of comparison. In Table II, the composition of each system and the nomenclature adopted in this work are summarized.

Moreover, it is worth noting that in the PEgMA/NPOSS nanocomposite, POSS loading is such that there is optimum nanoparticles concentration to have high efficiency of the reaction between maleic anhydride and POSS functionality. ${ }^{22}$

The samples used for the characterization were prepared from a pelletized material using a Carver hydraulic laboratory press (Wabash, IN, USA); the pellets were preheated for $2 \mathrm{~min}$ and then pressed at the temperature of $180^{\circ} \mathrm{C}$ under pressure of nine bars for $5 \mathrm{~min}$.

Disk-shaped samples were used for the rheological characterization, which was performed using a plate-plate rotational 
TABLE II

\begin{tabular}{llc} 
Compositions of Investigated Samples & \multicolumn{2}{c}{ Composition(wt\%) } \\
\hline Sample ID & PE & 100 \\
\hline PE & PE & 99.7 \\
PE/DCP & DCP & 0.3 \\
PE/1POSS & PE & 97 \\
PE/DCP/1POSS & POSS & 3 \\
& PE & 96.7 \\
PEgMA & DCP & 0.3 \\
PEgMA/NPOSS & 1POSS & 3 \\
& PEgMA & 100 \\
& PEgMA & 97 \\
& NPOSS & 3 \\
\hline
\end{tabular}

rheometer Ares G2, TA Instruments (Milan, Italy) operating at the frequency sweep mode at $180^{\circ} \mathrm{C}$. The strain amplitude was $\gamma=5 \%$, which in preliminary strain sweep experiments proved to be low enough to be in the linear viscoelastic region. Values of complex viscosity $\left(\eta^{*}\right)$, storage modulus $\left(G^{\prime}\right)$, and loss modulus $\left(G^{\prime \prime}\right)$ were recorded for all samples in the frequency $(\omega)$ range $0.01-100 \mathrm{rad} / \mathrm{s}$.

The Fourier transform infrared (FTIR) spectra were recorded on a Perkin-Elmer Spectrum One spectrometer (Waltham, Massachusetts, USA) in the $4000-400 \mathrm{~cm}^{-1}$ wave number range at room temperature. Sixteen scans with a resolution of $4 \mathrm{~cm}^{-1}$ were performed on film samples for each spectrum. POSS spectra were obtained analyzing a disk of KBr-POSS (4 wt $\%$ POSS). The FTIR spectra of the investigated nanocomposites are normalized with respect to the PE reference peak at $2019 \mathrm{~cm}^{-1}$. $^{24}$

Soxhlet extractions were performed for $60 \mathrm{~h}$ in xylene, which is a good solvent for polymer matrix and POSS nanofillers too. After the extraction, the samples were left to dry for $72 \mathrm{~h}$, weighted a first time and weighted again after $24 \mathrm{~h}$ to ensure solvent evaporation.

Micrographs of cryogenic fractured surfaces of all samples were obtained by a scanning electron microscope by a Philips ESEM XL30 (Amsterdam, The Netherlands) equipment. Voltage and work distance used are reported in the micrographs.

X-ray diffraction (XRD) patterns were obtained on an Empyrean Series 2 (panalytical) diffractometer (Frankfurt am Main, Germany) using $\mathrm{Cu} \mathrm{K} \alpha$ radiation at wavelength $\lambda=$ $0.1542 \mathrm{~nm}$. The spectra of the sample films and neat POSS powders were recorded in the range $3-30 \mathrm{deg}$ with a step size $=0.025$ and a scanning rate $=60 \mathrm{~s} / \mathrm{step}$.

Calorimetric analysis was performed on a Perkin-Elmer DSC7 calorimeter (Billerica, Massachusetts, USA); inert atmosphere $\left(\mathrm{N}_{2}\right)$ was used in the measurements. The samples were first subject to a heating cycle from 30 to $180^{\circ} \mathrm{C}$ at $10^{\circ} \mathrm{C} / \mathrm{min}$ to erase thermal history, then cooled back to $30^{\circ} \mathrm{C}$ at $40^{\circ} \mathrm{C} / \mathrm{min}$, heated a second time at $5^{\circ} \mathrm{C} / \mathrm{min}$, and cooled once again. Melting temperature and enthalpy were evaluated from the first and the second heating scans.

Rectangular samples were obtained from compressionmolded sheets and used for the mechanical characterization, which was performed on a Zwick Roell Z005 dynamometer (Ulm, Germany) according to ASTM D882 standard. Elastic modulus $(E)$, tensile strength (TS), and elongation at break (EB) values were calculated with a reproducibility of $\pm 5 \%$.

\section{Results and Discussion}

The melt rheological properties of the hybrids and of reference samples are shown in Fig. 1. The complex viscosity $\eta^{*}$, elastic $\left(G^{\prime}\right)$, and loss $\left(G^{\prime \prime}\right)$ modulus versus frequency for the PEgMA/NPOSS hybrid (reported on the left part in Fig. 1) are quite different from that of the neat PEgMA polymeric matrix and highlight that a rheological transition from liquid-like to solid-like occurring as a result of chemical reaction between the maleic groups of PEgMA and the amine functionality of NPOSS. The complex viscosity values at low frequency for the PEgMA/NPOSS system are significantly greater than those of the neat matrix. Also, the shear-thinning behavior for the filled system is more pronounced with respect to that of the PEgMA. Indeed, the PEgMA/NPOSS hybrid shows a rheological behavior similar to that shown by a cross-linked polymer, i.e., an almost frequency-independent elastic modulus $G^{\prime}(\omega)$ and loss modulus $G^{\prime \prime}(\omega)$ in all investigated frequency regions (solid-like behavior), and $G />G^{\prime \prime}$, typical of rubber-like linear viscoelastic behavior.

These results can be mainly explained considering:

1. the grafting on the polymeric backbone of NPOSS molecules that became an anchor point for the matrix because of strong nanofiller/matrix interactions ${ }^{21}$,

2. a filler-filler network arising from the local interactions between grafted NPOSS cages themselves that have substituent with same polarity and also have the possibility of multiple Van der Waals interactions intra- and intermolecularly for each POSS cage ${ }^{25}$ (see Fig. 2).

Besides, owing to the presence of two reactive amines for each reactive group of the NPOSS molecules, it cannot be excluded that the nanofiller may act as a cross-linking point.

The analysis of the trend of viscosity versus frequency for the PE/1POSS suggests that unbound POSS molecules act as a plasticizer of $\mathrm{PE}$, thus increasing free volume in the melt; this last effect leads to lower $G^{\prime}$ and $G^{\prime \prime}$ with respect to the neat matrix. ${ }^{9}$ The DCP loading causes cross-linking and branching of the polymer matrix as deducible by the viscosity values and $G^{\prime}$ slope increase in the low-frequency range as compared to the neat polymer matrix. Finally, comparing the rheological behavior of the PE/DCP/1POSS hybrid and PE matrix, it can be inferred that in the loaded system two opposing effects occur: The physically dispersed unreacted POSS leading to lower viscosity and moduli values and, on the other hand, grafted POSS molecules, affecting polymer chain mobility, result in higher viscosity and moduli. ${ }^{26}$

It is worth noting that hybrids require a sufficient amount of reacted POSS with the host polymer chains to change rheological behavior from liquid-like to solid-like. It has been shown in the literature that if the length scale between successive POSS attachments is comparable to that of a single POSS molecule intrachain POSS-POSS interactions may be predominant and act like a physical cross-link. ${ }^{27}$ Therefore, performed rheological characterizations establish that PEgMA/NPOSS hybrid contains high amounts of grafted POSS whereas in PE/DCP/1POSS hybrid, grafted POSS molecules are surrounded mainly by segments of the polymer chain, so that the intrachain POSS-POSS 

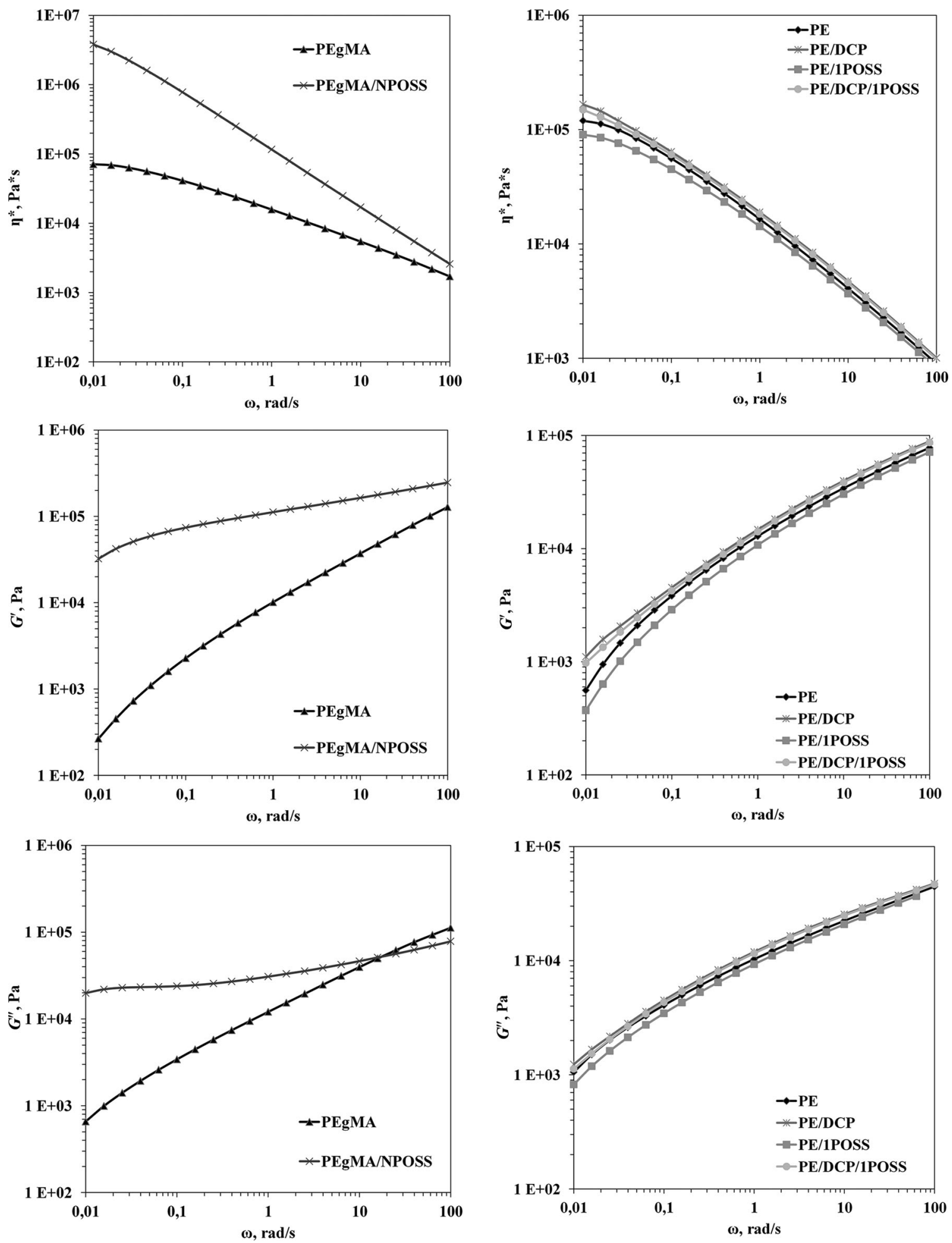

FIGURE 1. Viscosity curves, $\eta^{\star}$; storage, $G^{\prime}$ and loss, $G^{\prime \prime}$ moduli of all investigated samples.

interactions are shielded and do not significantly influence the polymer dynamics in the melt.

To confirm the formation of POSS-grafted hybrids through reactive processing, infrared spectroscopy analysis is performed and in Figs. 3-5 FTIR spectra are shown. FTIR spectra of pristine used POSS powders are reported in Fig. 3 for the sake of comparison, along with an enlargement in the range 1950-1550 $\mathrm{cm}^{-1}$, of interest for the corresponding polymeric investigated hybrids. In the 1POSS spectrum, a definite peak at $1636 \mathrm{~cm}^{-1}$ is noticeable, resulting from the vibration of the allyl double bond of 


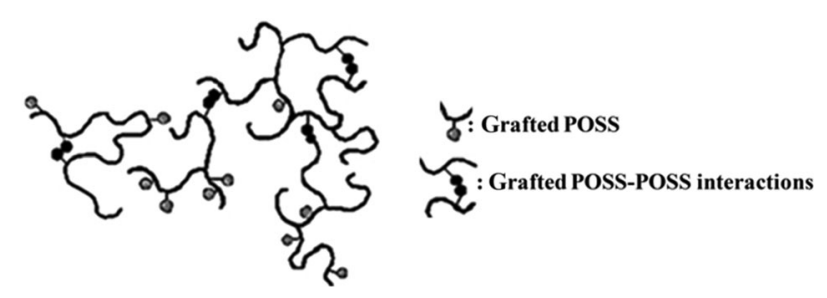

FIGURE 2. Interactions between grafted POSS.

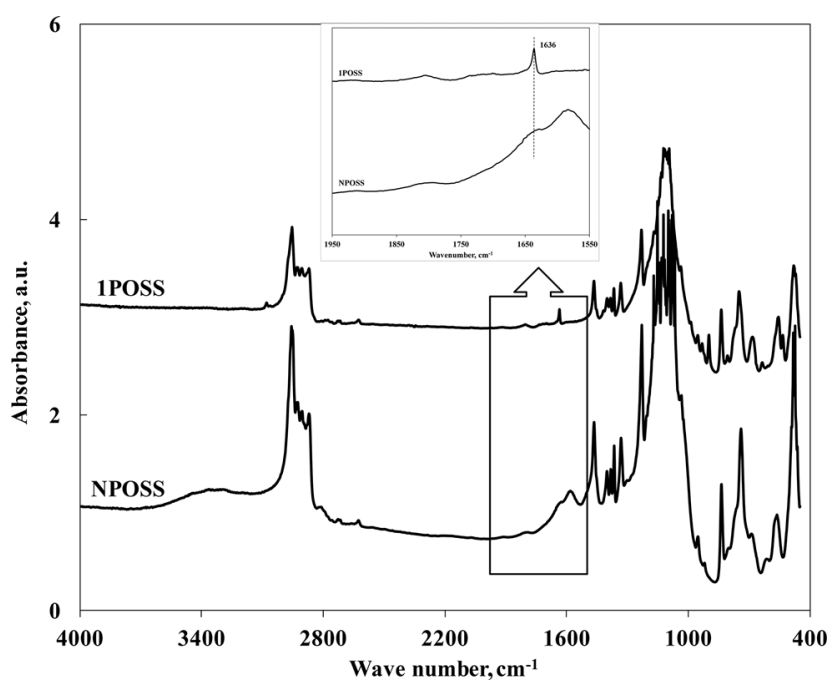

FIGURE 3. FTIR spectra of NPOSS and 1POSS on KBr disk; in the inset an enlargement in the range $1950-1550 \mathrm{~cm}^{-1}$.

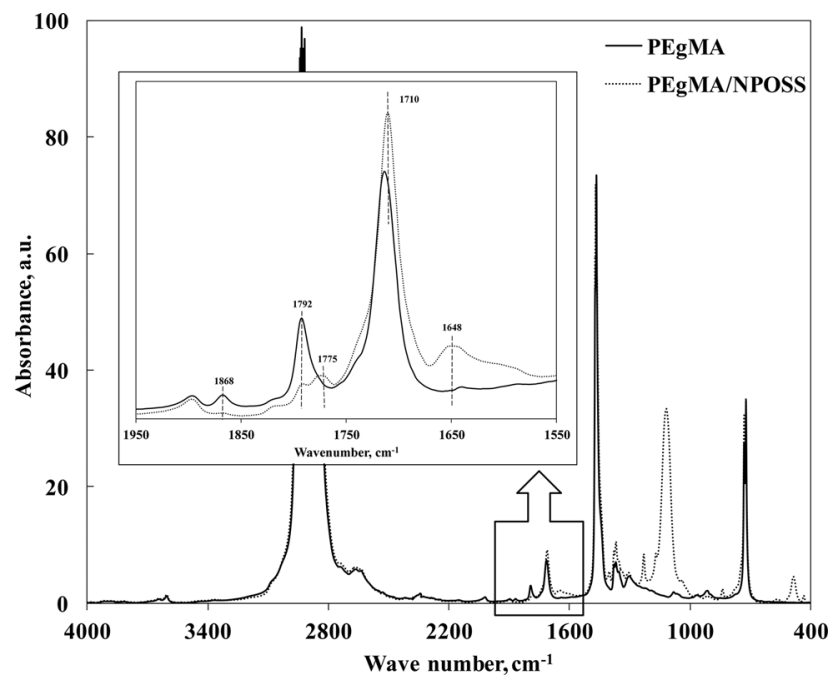

FIGURE 4. FTIR spectra of PEgMA and PEgMA/NPOSS; in the inset an enlargement in the range $1950-1550 \mathrm{~cm}^{-1}$.

the functional group. The $\mathrm{N}-\mathrm{H}$ bending vibration of the NPOSS functional group is observed in the $1650-1580 \mathrm{~cm}^{-1}$ region of the spectrum.

The PEgMA spectrum (Fig. 4) shows absorption bands centered at 1868 and $1792 \mathrm{~cm}^{-1}$ assigned to asymmetric and symmetric stretching of grafted maleic anhydride carbonyl groups, respectively, whereas the one at $1713 \mathrm{~cm}^{-1}$ corresponds to

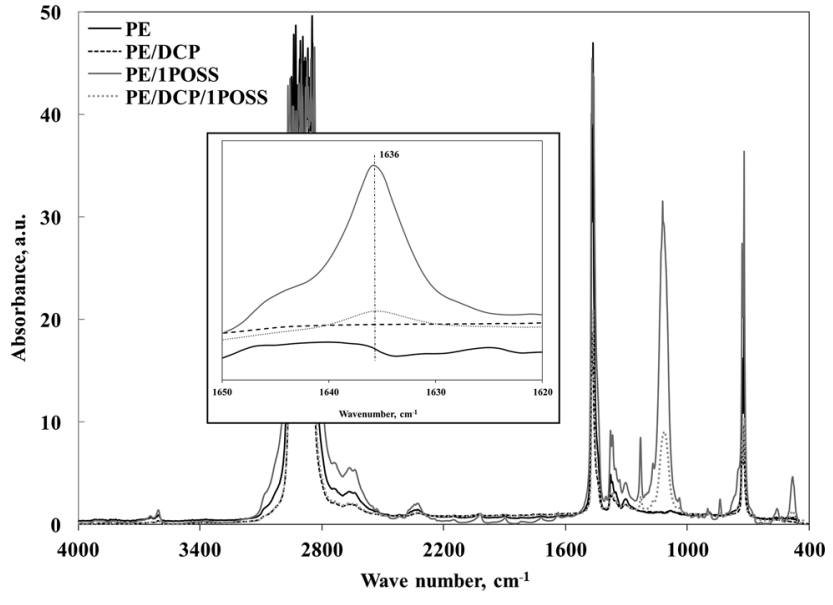

FIGURE 5. FTIR spectra of PE, PE/DCP, PE/1POSS, and $\mathrm{PE} / \mathrm{DCP} / 1 \mathrm{POSS}$; in the inset an enlargement in the range $1620-1650 \mathrm{~cm}^{-1}$.

anhydride carboxylic groups stretching. As clearly visible in the portion of amplificatory FTIR spectra of PEgMA/NPOSS reported in the inset of Fig. 4, the signals at 1868 and $1792 \mathrm{~cm}^{-1}$ are still present, even if with significantly lower intensity, indicating the presence of a small amount of nonreacted maleic anhydride. However, in this last spectrum, new absorptions bands at 1775 and $1710 \mathrm{~cm}^{-1}$ appear, suggesting the formation of imide groups because of the reaction between functional groups of the polymeric matrix and primary amine of NPOSS molecules. ${ }^{22}$ Besides, in PE- $g$-MA/NPOSS spectrum, a characteristic of the amide group peak at $1648 \mathrm{~cm}^{-1}$ arises ${ }^{28}$ due to the competing reaction of secondary amine of NPOSS and anhydride maleic groups. Besides, the presence of the amide group might be an indication that the PEgMA/NPOSS is chemically cross-linked by a reaction with the difunctional amino-POSS.

These findings confirm that NPOSS molecules were successfully grafted onto the polymeric backbone, resulting in the hybrid nanocomposite also in accordance with the abovementioned rheological investigation .

The peak at $1636 \mathrm{~cm}^{-1}$ is well visible in the PE/1POSS spectrum, whereas its intensity decreases in the spectrum of the PE/DCP/1POSS hybrid (see Fig. 5). The signal attributed to the double bond of the POSS structure in the POSS-loaded systems suggests the presence of 1POSS only physically dispersed in the polymeric matrix, although the lower intensity of the signal can be related to the 1POSS ability to react with the polymeric matrix in DCP presence. It was reported in the literature that DCP acts as an initiator for grafting and cross-linking reaction in the presence of POSS molecules bearing a double bond, in particular activating the unsaturation of the reactive functional group of POSS..$^{29,30}$ Indeed, it is generally understood that peroxide abstracts hydrogen atoms from the PE chain and these radicals react with the olefin on POSS. In our case, we have to consider the reactivity of the allylic hydrogen of the allyl POSS: The energy required for the formation of allylic radicals on the allyl POSS is lower than that for the formation of secondary radicals on the PE. Moreover, if allyl radicals are formed they could react with the allyl olefin of POSS to form dimers or oligomers which could still contain olefinic groups; this might also be a partial 
TABLE III

The Percentage of Retained Mass of All Investigated Samples After Soxhlet Extraction

\begin{tabular}{lc}
\hline Material & Retained Mass (\%) \\
\hline PE & 0.0 \\
PE/DCP & 3.7 \\
PE/1POSS & 0.0 \\
PE/DCP/1POSS & 6.8 \\
PEgMA & 0.0 \\
PEgMA/NPOSS & 40.3 \\
\hline
\end{tabular}

explanation for some of the unreacted allyl-POSS, as assessed from FTIR analysis, and aggregates seen in the SEM (see below).

Additionally, grafting of POSS molecules was also assessed by Soxhlet extraction in xylene; Table III shows the percentage of retained mass, calculated as (final mass, i.e., after extraction and solvent evaporation)/(initial mass, i.e., before solvent extraction $)^{*} 100$. Both neat matrices, i.e., PE and PEgMA, completely dissolved in the solvent as well as PE/1POSS nanocomposite, since the nanofiller is only physically dispersed within the matrix. The percentage of retained mass of PE/DCP is $3.7 \%$ as DCP causes cross-linking and branching of the polymer matrix and the percentage of retained mass increases for PE/DCP/1POSS due to the presence of grafted molecules too. The PEgMA/NPOSS system retains the $40 \%$ of initial mass, because of the amino-anhydride reaction between both amine groups of POSS and functional groups of polymeric chains. In particular, this result seems to suggest the formation of chemically cross-linked system, also according to the above-reported rheological analysis.

Morphological characterization was performed by means of SEM and XRD measurements. In Fig. 6, SEM micrographs, at two different magnifications, of all investigated systems are reported. In the PEgMA/NPOSS micrograph (see Fig. 6b), POSS aggregates at the micrometric level are not detectable; so the analysis suggests a POSS dispersion on the nanometer level in this hybrid and highlights that the effective grafting of POSS cages on maleic anhydride-functionalized PE chains enhances nanofiller dispersion. The DCP presence causes cross-linking and branching but also chain scission and production of small pores in the polymeric matrix that are clearly visible in the micrographs as shown in Fig. $6 \mathrm{~d}$.

For the physical-blended nanocomposite PE/1POSS, it is possible to note that 1POSS particles agglomerate and crystallites are dispersed in the PE matrix (see Fig. 6e). The phase separation occurs because the Van der Waals interactions between POSS molecules and polymer segments may not be sufficient to avoid POSS agglomeration and because the used concentration of POSS may exceed the critical concentration after which the POSS tends to remain as separate domains. ${ }^{31}$

The 1POSS molecules are more uniformly dispersed in the PE/DCP/1POSS hybrid obtained by the reactive melt mixing, although residual micrometer-sized agglomerates and crystallites are still present in the polymer matrix (Fig. 6f). The micrograph of this last sample, however, does not show pores of the matrix, suggesting that DCP action is predominant in activating double bonds of POSS molecules and specifically in activating allyl groups at the surface of POSS aggregates.
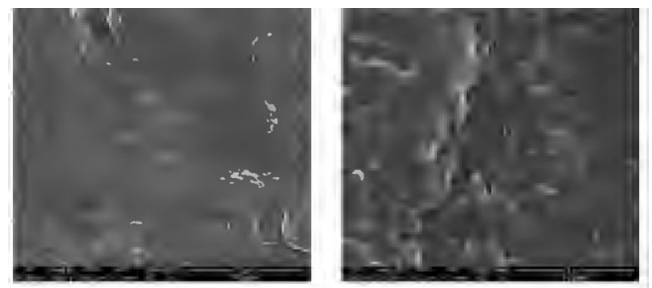

a
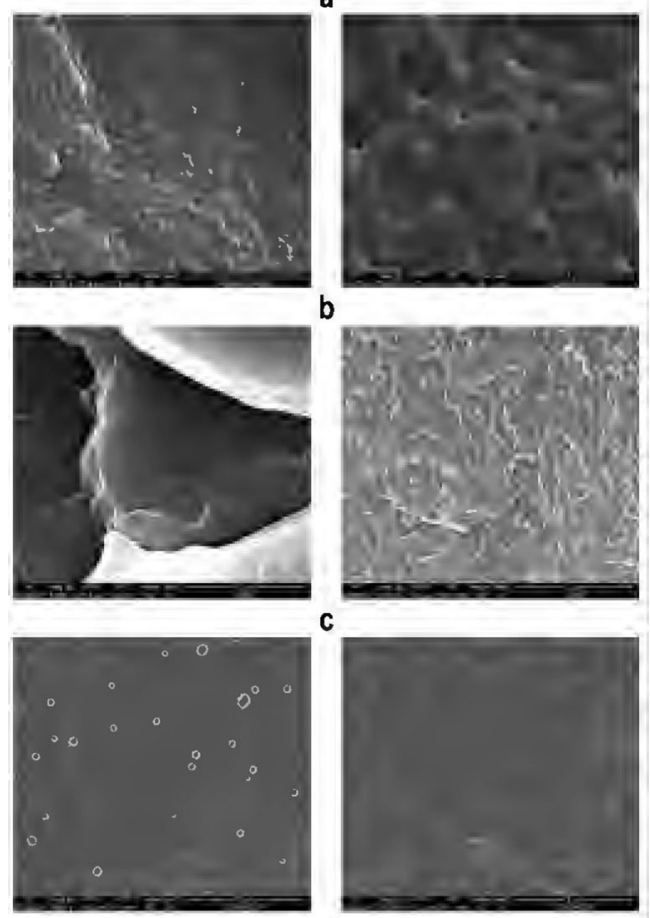

d
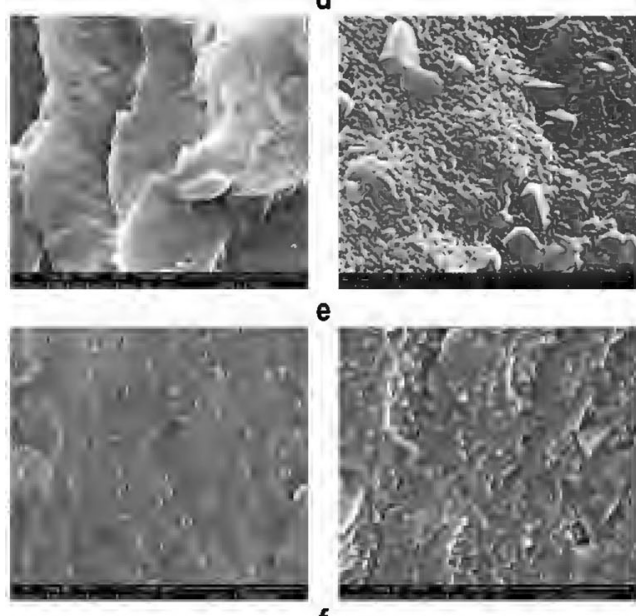

FIGURE 6. SEM micrographs at two different magnifications of (a) PEgMA, (b) PEgMA/NPOSS, (c) PE, (d) PE/DCP, (e)PE/1POSS, and (f) PE/DCP/1POSS.

Diffraction patterns of investigated hybrids and reference samples, as well as of neat nanofillers, are shown in Figs. 7a and $7 \mathrm{~b}$. PEgMA shows typical XRD crystalline signals assigned to the PE orthorhombic crystal, whereas neat NPOSS displays the principal diffraction peak at $2 \theta=8 \mathrm{deg}$ (see Fig. 7a). The XRD pattern for PEgMA/NPOSS, reported in Fig. 7a, is very similar 

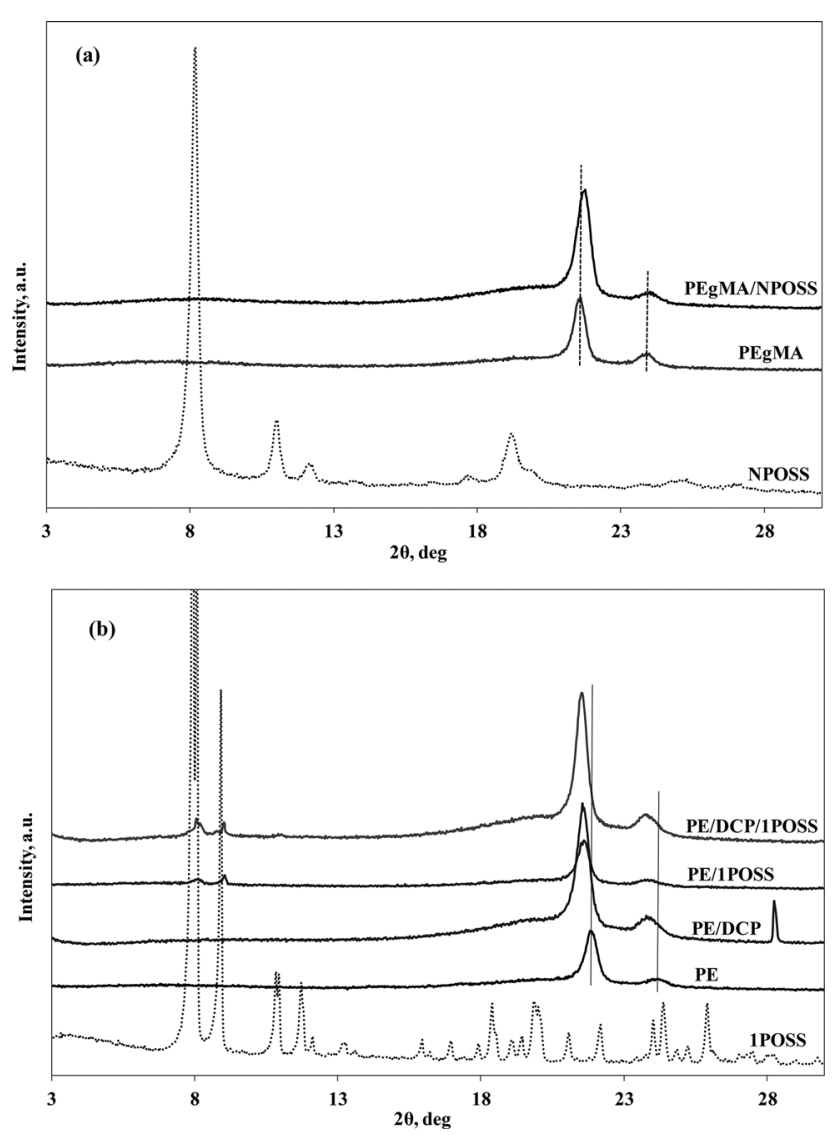

FIGURE 7. XRD patterns of (a) PE, PE/DCP, PE/1POSS, PE/DCP/1POSS and (b) PEgMA and PEgMA/NPOSS.

to neat PEgMA, and no signals related to NPOSS crystalline structures were detected. These findings suggest that NPOSS molecules are highly dispersed within the matrix by means of grafting reactions.

As visible in Fig. 7b, diffractogram of PE shows XRD typical crystalline peaks at $21.8(110)$ and $24.1(200)$ related to the orthorhombic unit cell. X-ray spectra of PE/1POSS display reflections characteristic of 1 POSS crystals in $2 \theta$ range $7^{\circ}-9^{\circ}$, signifying the presence of POSS crystallites in the nonreactive system. POSS crystalline structures were also detected in the PE/DCP/1POSS system, in accordance with results obtained from SEM analysis.
Moreover, it is noticed that DCP and/or 1POSS molecules contents, shift (110) and (200) crystalline peaks toward lower $2 \theta$ values (see Fig. $7 \mathrm{~b}$ ) likely because of the fillers' influence on the amorphous interlayer region. Cross-linked or POSSgrafted PE segments could not be incorporated into crystals and may rearrange themselves into interlamellar amorphous layers.

The SEM and X-ray morphological analysis confirm that a good dispersion is related to the formation of hybrid system with a good level of grafted nanofillers and that the chemical reactions between POSS molecules and the polymer matrix are useful to achieve nanostructured materials.

Table IV summarizes the results obtained from DSC analysis, and in Figs. 8 and 9 the thermograms of all investigated samples at first and second heating scans, respectively, are reported. From the first and second heating scan data, no significant influence of additives on the melt temperatures of PE and PEgMA was recorded. It worth noting that the filled systems based on PE, and even more on PEgMA, approach to have a lower onset temperature with respect to those of the neat matrices, in accordance with X-ray analysis (see Table IV). In Figs. 8 and 9, it is visible that the maleated PE sample shows multiple broad peaks and grafted NPOSS on maleic anhydride-functionalized PE mostly influences the lower melt temperature. The analysis of the fusion enthalpy data (see Table IV) suggests that POSS molecules grafting, as well as the cross-linking in DCP presence hinders the folding of macromolecular chains whereas the physically dispersed aggregated POSS molecules can act as a nucleating agent for PE crystallinity. ${ }^{32,33}$ However, the thermal behavior can be related to the degree of POSS dispersion within the polymer matrix: Lower crystallization is due to highly dispersed fillers, whereas crystal nucleation effects are predominant at low extent of dispersion. From DSC studies, it can also be concluded that the grafting is more effective in PEgMA/NPOSS hybrid than in PE/DCP/1POSS since with increasing grafted POSS molecules amount, the decrease in crystallinity is more significant.

The Young's modulus (E), TS at break, and EB for all investigated samples are reported in Table V. For all loaded samples, no significant changes in TS were recorded with respect to the neat correspondent matrices. The addition of unbounded 1POSS to PE produces an increase in the EB and a decrease in $E$, as it is generally found for plasticized polymeric systems. Conversely, cross-linking due to DCP loading results in a higher elastic modulus and lower EB compared to the neat matrix. PE/DCP/1POSS hybrid shows moderate difference with respect to the

TABLE IV

Thermal Properties of PE, PE/DCP, PE/1POSS, PE/DCP/1POSS, PEgMA, and PEgMA/NPOSS

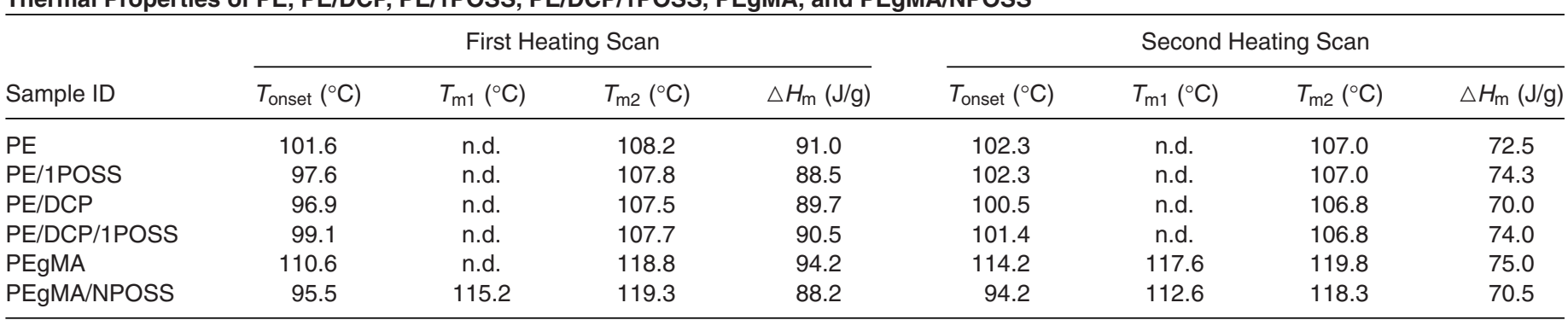



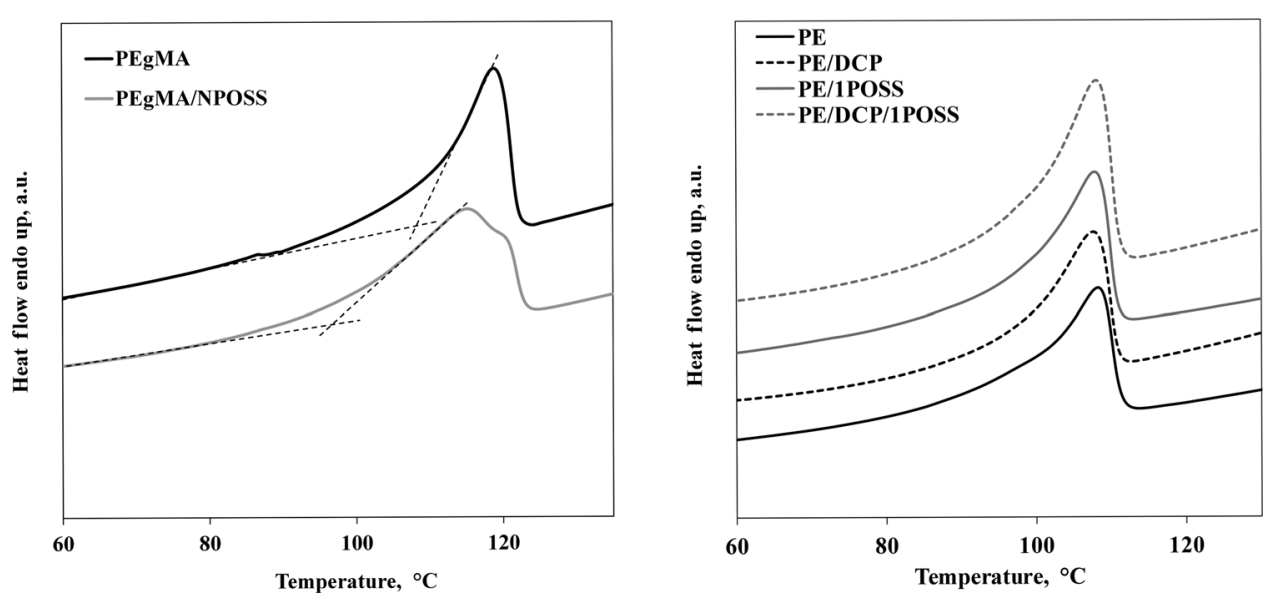

FIGURE 8. DSC traces at first heating scan of all investigated samples.
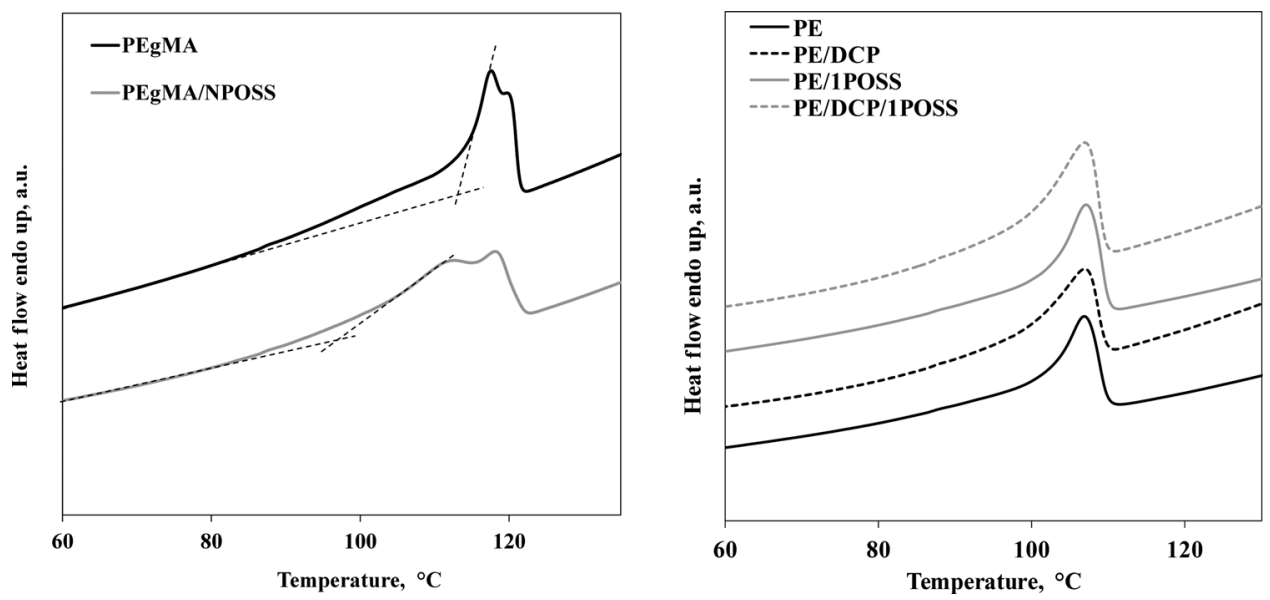

FIGURE 9. DSC traces at second heating scan of all investigated samples.

TABLE V Mechanical Properties of PE, PE/DCP, PE/1POSS, PE/DCP/1POSS, PEgMA, and PEgMA/NPOSS

\begin{tabular}{lccc}
\hline Sample ID & E (MPa) & TS (MPa) & EB (\%) \\
\hline PE & $250 \pm 12$ & $11.3 \pm 0.6$ & $550 \pm 27$ \\
PE/1POSS & $232 \pm 12$ & $11.1 \pm 0.6$ & $580 \pm 29$ \\
PE/DCP & $272 \pm 14$ & $11.0 \pm 0.6$ & $490 \pm 24$ \\
PE/DCP/1POSS & $244 \pm 12$ & $11.2 \pm 0.6$ & $520 \pm 26$ \\
PEgMA & $268 \pm 13$ & $19.1 \pm 1.0$ & $610 \pm 3$ \\
PEgMA/NPOSS & $274 \pm 14$ & $18.8 \pm 0.9$ & $425 \pm 21$ \\
\hline
\end{tabular}

mechanical performance of PE because it suffers contrasting effects due to 1POSS molecules presence both chemically bounded to polymeric chain and physically dispersed within the matrix together. The mechanical properties of PEgMA/NPOSS hybrid show a slight increase in $E$ and a severe reduction in EB. Grafted POSS to the polymeric backbone hinders the chain motion of macromolecules, which would improve the modulus; on the other hand, changes in crystallinity leading to a decrease in the elastic modulus in the hybrid would hide and dampen the intrinsic reinforcement action of the POSS particles.

\section{Conclusions}

The improvement in the properties depends on the quality and homogeneity of the POSS dispersion in the polymer and on POSS-polymer interactions. Grafting of POSS molecules plays a fundamental role in determining the dispersion degree in the resulting polymeric hybrids. Indeed, in this study, it was shown that the Van der Waals interactions between POSS molecules and polymer segments may not be sufficient to avoid POSS agglomeration, whereas an efficient grafting leads to the formulation of homogeneous POSS polymer-based hybrids. In particular, two different grafting approaches, by one-step reactive melt mixing based on the amino-maleic reaction and double bonds triggered in DCP presence, were analyzed. The results revealed that different amounts of grafted POSS affect differently the properties of the nanocomposite: If the grafting is not efficient, as for PE/DCP/1POSS, the resulting hybrid still contains POSS agglomerates and crystallites. Moreover, the interactions between grafted 1POSS are negligible whereas the predominant interactions between physically dispersed nanoparticles do not significantly influence the viscoelastic response of PE matrix 
and the mechanical properties in the solid state. The grafting of NPOSS molecules in PEgMA was proved to be efficient because of the high efficiency of the amine-anhydride reaction in the molten state. The grafting helps the POSS dispersion in the polymeric matrix; moreover, a solid-like behavior for the hybrid melt is recorded mainly due to the formation, within the matrix, of a network which then affects the mechanical performance.

\section{References}

1. Sanchez, C.; Julian, B.; Belleville, P.; Popall, M. J Mater Chem 2005, 15, 3559.

2. Camargo, P. H. C.; Satyanarayana, K. G.; Wypych, F. Mater Res 2009, 12, 1.

3. Pielichowski, K.; Njuguna, J.; Janowski, B. J Adv Polym Sci 2006, 201, 225.

4. Schwab, J. J.; Lichtenhan, J. D. Appl Organomet Chem 1998, 12, 707.

5. Lee, K. S.; Chang, Y. W. Polym Int 2013, 62, 64.

6. Zhao, Y.; Schiraldi, D. A. Polymer 2005, 46, 11640.

7. Wu, J.; Mather, P. T. J. Macromol Sci, Part C: Polym Rev 2009, 49, 25.

8. Moore, B. M.; Ramirez, S. M.; Yandek, G. R.; Haddad, T. S.; Mabry, J. M. J Organomet Chem 2011, 696, 2676.

9. Dintcheva, N. Tz.; Morici, E.; Arrigo, R.; La Mantia, F. P.; Malatesta, V.; Schwab, J. J. eXPRESS Polym Lett 2012, 6, 561.

10. Kodal, M.; Sirin, H.; Ozkoc, G. Polym Eng Sci 2014, 54, 264.

11. Dintcheva, N. Tz.; Morici, E.; Arrigo, R.; La Mantia, F. P. Polym J 2014, 46, 160.

12. Morici, E.; Arrigo, R.; Dintcheva, N. Tz. J Polym Eng 2015, 35, 329.
13. Dintcheva, N. Tz.; Morici, E.; Arrigo, R.; La Mantia, F. P.; Malatesta, V.; Schwab, J. J. Polym Degrad Stab 2012, 97, 2313.

14. Kuo, S. W.; Chang, F. C. Prog Polym Sci 2011, 36, 1649.

15. Jiao, J.; Lv, P.; Wang, L.; Cai, Y.; Liu, P. Polym Eng Sci 2015, 55, 565.

16. Zheng, L.; Farris, R. J.; Coughlin, E. B. Macromolecules 2001, 34, 8034.

17. Yuanchenga, Q.; Yutieb, B.; Hongbob, R.; Fanghuab, Z.; Meiming, L.; Lin, Z. Chin J Chem 2010, 28, 2527.

18. Zhou, Z.; Cui, L.; Zhang, Y.; Zhang, Y.; Yin, N. Eur Polym J 2008, 44, 3057.

19. Chevigny, C.; Dalmas, F.; Di Cola, E.; Gigmes, D.; Bertin, D.; Bouè, F.; Jestin, J. Macromolecules 2011, 44, 122.

20. Chen, P.; Huang, X.; Zhang, Q.; Xi, K.; Jia, X. Polymer 2013, 54, 1091.

21. Zhou, Z.; Zhang, Y.; Zhang, Y.; Yin, N. J Polym Sci, Part B: Polym Phys 2008, 46,526 .

22. Fina, A.; Tabuani, D.; Peijs, T.; Camino, G. Polymer 2009, 50, 218.

23. Kumar, S.; Butola, B. S.; Joshi, M. Fibers Polym 2013, 14, 428.

24. Dintcheva, N. Tz.; Al-Malaika, S.; La Mantia, F. P. Polym Degrad Stab 2009, 94, 1571.

25. Romo-Uribe, A.; Mather, P. T.; Haddad, T. S.; Lichtenhan, J. D. J Polym Sci, Part B: Polym Phys 1998, 36, 1857.

26. Grala, M.; Bartczak, Z. Polym Eng Sci 2015, 55, 2058.

27. Lee, A.; Xiao, J.; Feher, F. J. Macromolecules 2005, 38, 438.

28. Silverstein, R. M.; Webster, F. X.; Kiemle, D. J. Spectrometric identification of inorganic compounds; John Wiley \& Sons: New York, 2005; pp. 72-126.

29. Kumar, S.; Butola, B. S.; Joshi, M. Fibers Polym 2013, 14, 428.

30. Wu, J.; Wu, Z. L.; Yang, H.; Zheng, Q. RSC Adv 2014, 4, 44030

31. Wu, J.; Haddad, T. S.; Gyeong-Man, K.; Mather, P. T. Macromolecules 2007, 40, 544.

32. Pracella, M.; Chionna, D.; Fina, A.; Tabuani, D.; Frache, A.; Camino, G. Macromol Symp 2006, 234, 59.

33. Chen, J. H.; Yao, B. Z.; Su, W. B.; Yang, Y. B. Polymer 2007, 48, 1756. 